DOI 10.37882/2500-3682.2020.09.17

\title{
ПАТОЛОГИЯ КАК ИНДИКАТОР НОРМЫ
}

\section{EPISTEMOLOGY: PATHOLOGY AS AN NORM' INDICATOR}

\section{N. Tetenkov}

Summary: The article discusses one of the conceptual issues of epistemology: the possibility of cognizing the norm through the pathology. The relevance of the research topic is due to the fact that in the modern philosophy the problem of "pathology", as a way of cognizing the "norm", is considered mainly within the framework of the classical approaches by 0 . Comte, J.F. Brusset, as well as by K. Bernard. At the same time, modern supporters of neo-positivism do not take into account the specifics of the ideas about the "pathology" and "norm" by the classics themselves, since the views of J.F. Brousset and K. Bernard have fundamental differences from the generalizations of 0 . Comte, who extrapolated the dichotomy "norm-pathology" to social processes.

The purpose of the article is to more fully consider modern theses about "pathology" as a way of cognizing the "norm" within the epistemology framework. The article objectives are: to analyze modern scientific literature; to identify the main positions in classical approaches in relation to "norm" and "pathology" as epistemological categories, as well as in a critical analysis of some postulates by modern researchers. The article is debatable.

The research methodology is based on a systematic approach and includes a group of general scientific methods (analysis, synthesis, deduction, induction); as well as a group of special methods: content analysis of scientific literature on the research topic, retrospective and epistemological analysis. The author of the article comes to the conclusion that philosophical constructions, regarding the possibilities of indicating the norm through pathology, have a number of logical and epistemological limitations that must be eliminated by analyzing the state of the subject of cognition.

Keywords: epistemology, norm, pathology, epistemological categories, neo-positivism.

\author{
Тетенков Николай Борисович \\ К.ф.н., дочент, Северный (Арктический) федеральный \\ университет имени М.В. Ломоносова, \\ tenibo@yandex.ru
}

Аннотация: В статье рассматривается один из концептуальных вопросов эпистемологии: возможно ли познание нормы с помощью патологии. Актуальность темы исследования обусловлена тем, что в современной философии познания проблематика «патологии» как способа познания «нормы» рассматривается в основном в рамках классических подходов 0. Конта, Ж.Ф. Бруссе, а также К. Бернара, при этом современными сторонниками неопозитивизма не учитывается специфика представлений о «патологии» и «норме» самих классиков, поскольку взгляды Ж.Ф. Бруссе и К. Бернара имеют принципиальные отличия от обобщений 0. Конта, экстраполировавшего дихотомию «норма-патология» на социальные процессы.

Цель статьи заключается в том, чтобы более полно рассмотреть современные тезисы 0 «патологии» как способе познания «нормы» в рамках эпистемологии. Задачи статьи заключаются в проведении анализа современной научной литературы; выявление основных позиций в классических подходах в отношении «нормы» и «патологии» как эпистемологических категорий, а также в критическом анализе некоторых постулатов современных исследователей. Статья носит дискуссионный характер.

Методология исследования основана на системном подходе и включает в себя группу общенаучных методов (анализ, синтез, дедукция, индукция); а также группу специальных методов: контент-анализ научной литературы по теме исследования, ретроспективный и гносеологический анализ. Автор статьи приходит к выводу о том, что философские построения относительно возможностей индикации нормы через патологию имеют ряд логических и эпистемологических ограничений, которые необходимо устранить с помощью анализа состояния субъекта познания.

Ключевые слова: эпистемология, норма, патология, гносеологические категории, неопозитивизм.

Историография исследуемой проблемы очень обширна и включает в себя труды как отечественных, так и зарубежных авторов. В числе первых необходимо отметить работы таких исследователей, как Е.И. Громова [1], А.Л. Дрозд [2], В.И. Жилин [3], Т.Д. Мустафазаде [4], В.В. Погорелов [5], чьи работы посвящены вопросам философии медицины в современной философии познания. Среди зарубежных авторов определенный интерес вызывают работы В. Фрере [6], Д. Левайна [7], С. Нормандина [8], В. О'Донахью [9], которые содержат некоторые критические суждения по вопросу о патологии как способу познания нормы. Подробный разбор тезисов О. Конта по вопросу патологии и нормы дается в работах таких авторов, как Ю.С. Парк, Л. Конге, Л.Р. Артино [10], 
М. Пикеринг [11], а также Б. С. Святчак и А.И. Таубер [12].

В целом, анализ научной литературы по теме исследования позволяет заключить, что в течение практически всего XIX века сущность нормальных и патологических витальных явлений, которые, по-видимому, были настолько разными, с учетом противоположных ценностей человеческого опыта, стала своего рода научной догмой, распространение которой в области философии и психологии, по-видимому, было продиктовано авторитетом биологов и медиков. Эта догма была изложена во французской философии познания основоположником позитивизма О. Контом и медиком-практиком К. Бернаром, каждый из которых работал в совершенно разных направлениях: первый был философом-теоретиком, второй - физиологом практиком.

В частности, своих размышлениях О. Конт переходит от патологического к нормальному с целью умозрительного определения законов нормального; поскольку он заменяет биологические эксперименты - часто невыполнимые, особенно для человека, логическими построениями. Именно в трудах О. Конта утверждается идентичность нормального и патологического как приобретение знаний о нормальном [11, с. 343]. То есть, для О. Конта патология является логически обоснованным индикатором нормы. В своих исследованиях К. Бернард, напротив, переходит от нормального к патологическому с целью рационального действия, направленного на патологическое; поскольку именно в качестве основы неэмпирической терапии необходимы знания о болезнях (на основе физиологии). Идентичность нормального и патологического утверждается как сравнение по результату устранения патологического [12, с.16]. Таким образом, О. Конт выводит сущность патологии как чистой логической концепции, как индикатора нормы; в то время как К. Бернар пытается уточнить сущность патологии в отношении нормы в количественной, численной интерпретации.

Распространение идей О. Конта и К. Бернара в медицинских, научных и литературных кругах больше всего ощущается в области психологии.

В частности, некоторые авторы полагают, что при изучении психологии личности сон, безумие, бред, сомнамбулизм, галлюцинации предлагают гораздо более благоприятную область опыта, чем нормальное состояние [10, с.18].

Явления, которые в нормальном состоянии почти стерты из-за их незначительности, кажутся более ощутимыми в чрезвычайных кризисах, потому что они преувеличены [9, с.24].

В своей статье Д. Левайн отмечает, что «физик не изу- чает гальванизм в слабых количествах, встречающихся в природе, но увеличивает его с помощью экспериментов, чтобы его было легче изучать, хотя законы, изученные в этом преувеличенном состоянии, идентичны естественному состоянию, то есть норме» [7, с.678].

По мнению других авторов, «человеческая психология должна изучать безумие человечества, мечты и галлюцинации, которые можно найти на каждой странице истории человеческого духа» [8, с.500]. Вероятно, поэтому сторонники концепции О. Конта полагают, что «физиология и патология, как физическая, так и психологическая, не противопоставляются друг другу как две противоположности, а скорее, должны изучаться как две части одного целого» [11, с.350].

Предложенный французскими исследователями «патологический» метод стремится одновременно к чистому наблюдению и экспериментам. Это мощное средство исследования: «болезнь, по сути, является экспериментом самого тонкого порядка, установленным самой природой в очень точных обстоятельствах с помощью средств, недоступных человеческому умению, поскольку природа достигает недоступного» [8, с.496]. Интересно, что знаменитый немецкий философ, Ф. Ницше, заимствовал именно у К. Бернара идею о том, что «патологическое однородно с нормальным». Цитируя длинный отрывок о здоровье и болезни, взятый из трактата K. Бернара «Lec, ons sur la chaleur animale» («Лекции о жаре у животных»), Ф. Ницше формулирует следующее утверждение: ценность всех болезненных состояний в том, что они показывают нам под увеличительным стеклом определенные состояния, которые являются нормальными, но не всегда хорошо видны в нормальном состоянии» $[8$, с.498].

Примечательно, что и в своей позитивистской концепции О. Конт часто ссылается на афоризм Ф.Ж. Бруссе: «каждая модификация - естественная или искусственная - реального порядка касается только интенсивности соответствующих явлений; несмотря на различия в степени, явления всегда сохраняют одно и то же расположение; кроме того, любое изменение в реальной природе, то есть классе объекта признается противоречивым» [11, с.342]. Более того, по сути, вся позитивистская теория изменчивости явлений полностью сводится к этому универсальному принципу и является результатом систематического применения афоризма Ф.Ж. Бруссе.

Непосредственно полезный для изучения биологических проблем, этот принцип рассматривается большинством исследователей в качестве логической подготовки к аналогичным процедурам в любой науке. Социум, как коллективный организм, из-за высокой степени его сложности, имеет множество проблем. Они разнообразны и более часты, чем у отдельного организма. Поэтому 
некоторые современные авторы склонны распространять принцип Ф.Ж. Бруссе на все социальные процессы, в частности, активно он применяется для ограничения или совершенствования социологических законов $[12$, c.30].

Следует признать, что суть эксперимента по принципу Ф.Ж. Бруссе заключается не в искусственном вмешательстве исследователя в систему явления, которое он намеренно стремится нарушить, а скорее в сравнении между контрольным явлением и феноменом, измененным в отношении любого из его условий.

В соответствии с исключительно философским принципом Ф.Ж. Бруссе, который стал в XXI веке общей основой для позитивистской концепции патологии, патологическое состояние вовсе не является радикально отличным от физиологического состояния, в отношении которого - независимо от того, как его интерпретирует субъект познания, - оно может представлять собой лишь простое расширение, выходящее более или менее за пределы более высоких или более низких пределов вариации, свойственных каждому явлению нормального состояния [12, с.32].

Следовательно, каждая концепция патологии должна основываться на предварительном знании соответствующего нормального состояния. Однако с точки зрения эпистемологии, научное изучение патологических случаев становится необходимой фазой в общем поиске законов нормального состояния. Наблюдение за патологическими случаями предлагает многочисленные, подлинные преимущества для фактического экспериментального исследования. Переход от нормального к патологическому состоянию является более медленным и естественным в случае болезни, а возвращение к норме, когда оно происходит, самопроизвольно обеспечивает необходимое противодействие.

Согласно такому подходу к патологии как к индикатору изменений нормы, некоторые современные исследователи предлагают изучение аномалий и чудовищ: «тератологический подход» (изучение монстров) дополняет позитивистский патологический метод» [9, с.27].

При этом, на наш взгляд, ставшая классической концепция О. Конта, нуждается в некоторых уточнениях с позиции эпистемологии. Во-первых, поскольку мы не можем связать такого рода общие положения с какимлибо примером, мы не знаем, с какой точки зрения О. Конт утверждает, что патологическое явление всегда имеет свой аналог в физиологическом явлении, и что оно не является радикально новым.

Во-вторых, следует отметить, что, несмотря на взаимный характер разъяснения, достигнутого путем срав- нения нормального с патологическим и ассимиляции патологического и нормального, О. Конт неоднократно настаивает на необходимости определения нормального и пределов вариации нормальности, прежде чем методически исследовать патологические случаи. Строго говоря, знание нормальных явлений, основанное исключительно на наблюдениях, возможно и необходимо без знания патологии, особенно на основе экспериментов.

Однако, в тезисах О. Конта и его последователей обнаруживается серьезный пробел в том, что никто из них до сих пор не предоставит критерия, который позволил бы нам узнать, что такое нормальное явление. Нам остается сделать вывод, что в этом отношении О. Конт и его последователи ссылаются на обычную соответствующую концепцию, учитывая тот факт, что они используют понятия нормального состояния, физиологического состояния и естественного состояния как взаимозаменяемые понятия [6, с.45].

Более того, когда речь идет об определении границ патологических или, или согласно позитивистскому патологическому методу- экспериментальных нарушений, совместимых с существованием организмов, О. Конт отождествляет эти пределы с границами «гармонии различных влияний, как внешних, так и внутренних» $[6$, с.46]. В результате такой трактовки понятие нормального или физиологического, окончательно проясненного этим понятием гармонии, равнозначно качественному и поливалентному понятию, все еще более эстетическому и моральному, чем научному.

Что касается утверждения об идентичности нормальных и соответствующего патологических явлений, то столь же ясно, что основная цель его в том, чтобы отрицать качественное различие между этими двумя феноменами. По логике вещей, отрицание качественных различий должно привести к утверждению однородности, способной выражаться в количественном выражении. Однако, следует признать, что используемые сторонниками концепции О. Конта количественные термины, они все же имеют качественный аспект, при этом различия между нормальным и патологическим состоянием могут быть выражены в количественном выражении, поскольку только количество позволяет учитывать как однородность, так и вариацию. Ставя под сомнение эту двойную возможность, мы не собираемся недооценивать ни физиологию, ни патологию. В любом случае должно быть очевидно, что ни Ф.Ж. Бруссе, ни О. Конт не выполнили два требования, которые кажутся неотделимыми от попытки понять норму через патологию, а их последователи преуспели только в построении концептуальной иерархии патологических явлений, качественного устройства для обозначения состояния между двумя крайностями здоровья и болезни [2, с.62]. 
Постулируя логически, мы можем отметить следующее: совершенно правильно, что идентификация явлений, качественные различия которых считаются иллюзорными, принимает форму количественного определения. Маркировка, идентификация одного явления через исследование его противоположности не является кардинальной единицей измерения.

Более того, нестабильность и нерегулярность являются существенными характеристиками жизненных явлений, так что попытки втиснуть их в жесткие рамки метрических отношений искажают их природу [9, с.37]. Таким образом, фактическое понятие нормы зависит от возможности ее нарушения. С точки зрения эпистемологии, такое утверждение практически полностью лишено смысла, поскольку если норму нельзя познать без ее изменения, а норма в ее измененном состоянии необязательно является патологией, то объект познания теряет всякий смысл. Более того, такого рода абстрактные суждения позволяют развивать довольно опасные для общества идеи о том, что норма и патология - есть одно и то же состояние, и различаются они только в количественном отношении, в измеряемом отклонении «либо в уменьшение, либо в увеличение».

Необходимо понимать, что такого рода «ускользающий» объект как измененная норма, не являющаяся патологией, особенно применительно к социуму, а не к индивидуальному организму, предполагает отсутствие четких критериев в сознании самого субъекта познания. Иными словами, невозможно постичь то, что постоянно изменяется, но при этом остается неизменным. Таким образом, все перечисленные подходы к патологии как индикатору нормы снова сводятся к кантовской трактовке «вещь в себе», что окончательно нивелирует весь смысл дальнейших рассуждений о способе познания нормы через патологию.

Более того, неопозитивистские идеи, выраженные в работах Ю.С. Парк, Л. Конге, Л.Р. Артино [10], М. Пикеринга [11], а также Б.С. Святчака и А.И. Таубера [12] по вопросу познания нормы через патологию, приводят не только к отождествлению нормы и патологии, при их исключительно количественных различиях, но и к размыванию критериев, используемых для определения этих категорий.

На примере общества как особого социального организма со сложной и подверженной «болезням» организацией, можно говорить о том, что концепция О. Конта в ее трансформированном виде привела к тому, что в западных обществах происходит процесс прямой подмены понятий, когда норма интерпретируется как патология, а патология, напротив, возводится в статус нормы. Таким образом, можно констатировать, что ни в одном из современных исследований, посвященных роли па- тологии в анализе нормы, нет акцента на гносеологический аспект проблемы: познающий субъект должен, по крайней мере, осознавать себя познающим, чтобы интерпретировать собственное состояние.

Если рассматривать патологию только как количественный инструмент познания нормы, как это делается в неопозитивизме и в работах сторонников Ж.Ф. Бруссе и К. Бернара, то с позиций гносеологии получается, что современное общество как субъект познания теряет свою главную характеристику субъективности, не осознавая себя познающий субъектом.

Без понимания того, что происходит некое изменение, невозможно судить о норме с помощью патологии, поскольку при таком подходе теряется значение обоих понятий, а суждения перестают отвечать критериям логики. При искаженном сознании самого субъекта познания, фактически, невозможно доказать, происходит ли изменение или нет, существует ли сама норма или нет.

Таким образом, способ познания нормы с помощью исследования патологии требует коренного пересмотра в современной философии, с учетом того, что все классические концепции все-таки относились не к социальной философии, но к философии медицины и несут на себе более чем заметный отпечаток физиологии и биологии. При этом органические теории общества на современном этапе, сколь ни близки они по духу к физиологическим концепциям в медицине, все же не отвечают новому состоянию общества как субъекта познания.

Подводя итог всего сказанному выше, можно сделать следующие выводы:

1. классические теории познания нормы через исследование патологии фактически свели саму категорию «патология» к индикаторы количественных отклонений для нормы;

2. в современной историографии вопроса доминирует тенденция к переосмыслению концепций О. Конта, Ф.Ж. Бруссе и К. Бернара в рамках общей концепции постмодернизма, когда, по сути, сам смысл познания подменяется процессом хаотической деятельности познающего субъекта, не осознающего свой познавательный опыт;

3. патология в качестве способа познания нормы требует конкретизации не только как общая медицинская, но и как гносеологическая категория. Поэтому наиболее перспективным направлением дальнейших исследований в сфере соотношения категорий «норма» и «патология» в современной гносеологии, на наш взгляд, является кардинальный пересмотр классического описательного и терминологического аппарата, применяемого изначально в области философии медицины. 


\section{ЛИТЕРАТУРА}

1. Громова Е.И. «Другой» и концептуализация психической болезни // Вестник Московского университета. Серия 7. Философия. 2016. № 6. - С. 33-44.

2. Дрозд А.Л. Проблема болезни и адаптационных возможностей человека в зеркале философской рефлексии // Манускрипт. 2017. № 12. - С. 63-67.

3. Жилин В.И. Психология восприятия и эпистемология // Известия Саратовского университета. Новая серия. Серия Философия. Психология. Педагогика. 2014. № 4-1. - С.18-23.

4. Мустафазаде Д.Т. Философия и медицина: диалектика их взаимосвязи // Бюллетень медицинских интернет-конференций. 2014. № 11. - С. 11-23. ISSN 2224-6150.

5. Погорелов В.В. Соотношение естественнонаучного и гуманитарного подходов в современных исследованиях проблем здоровья человека и общества // Евразийский Союз Ученых. 2014. № 5-6. - С. 1-4.

6. Frere B. Positivism. Chapter in The Blackwell Encyclopedia of Sociology 2020. - P. 44-69. D0I: 10.1002/9781405165518.wbeosp061.pub2

7. Levine D. Auguste Comte and Positivism: The Essential Writings. Contemporary Sociology. 2019. Vol.47(6). - P. 677-678. D0I: 10.1177/0094306118805421cISBN: 0094-3061

8. Normandin S. Claude Bernard and An Introduction to the Study of Experimental Medicine: «Physical Vitalism,» Dialectic, and Epistemology. Journal of the History of Medicine and Allied Sciences. 2017. Vol. 62(4). - P. 495-528.

9. O'Donohue W. Epistemology and Logical Positivism. Chapter in the book Clinical Psychology and the Philosophy of Science. 2nd edition. 2019. - P.23-42.

10. Park Y.S., Konge L., Artino L.R. The Positivism Paradigm of Research. Academic Medicine. 2020. Vol. 95(5):1. - P. 17-26. D0l: 10.1097/ ACM.0000000000003093ISBN: 1040-2446

11. Pickering M. Auguste Comte and Positivism. In book: The Cambridge History of French Thought. 2019. - P. 342-352. D0I: 10.1017/9781316681572.040ISBN: 9781107163676

12. Swiatczak B., Tauber A.I. Philosophy of Immunology. Chapter in Stanford Encyclopedia of Philosophy. 2020. - P. 15-44. D0I: 10.1093/ 0so/9780190098353.003.00011SBN: 9780190098353

(с Тетенков Николай Борисович (tenibo@yandex.ru)

Журнал «Современная наука: актуальные проблемы теории и практики»

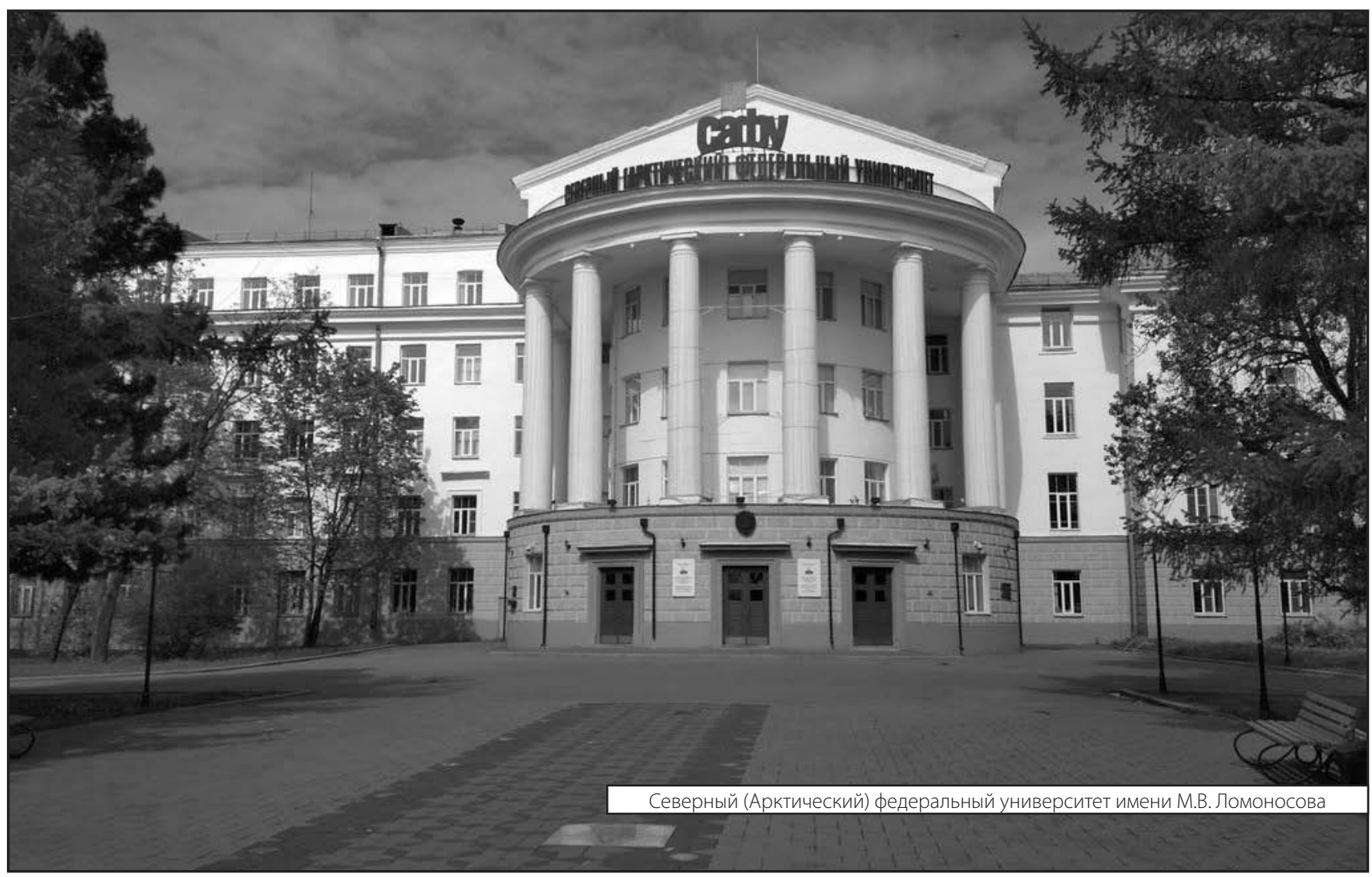

